Please send trade news information and illustrations to Arveen Bajaj at the $B D J$, Nature Publishing Group, The Macmillan Building, 4-6 Crinan Street, London N1 9XW.

Trade news is provided as a service to readers using text and images from the manufacturer, supplier or distributor and does not imply endorsement by the BDJ. Normal and prudent research should be exercised before purchase or use of any product mentioned.

\title{
Contouring composite New mouth cancer detection test
} \section{restorations}

The new CompoRoller from KerrHawe is a new instrument designed to give absolute control in the layering and contouring of direct composite restorations. The CompoRoller's unique rolling action contours composites quickly in thin and even layers, resulting in less time spent finishing and polishing. The non-stick rollers ensure bubble-free restorations with no 'pull-back' and allow you to place your composite in the thinnest sections. Made from smooth, medical grade thermoplastic elastomer the disposable rollers are available in two shapes, cylindrical for flat-surface contouring and a conical shape for occlusal surfaces, modelling and shaping margins.

Reader response number 50

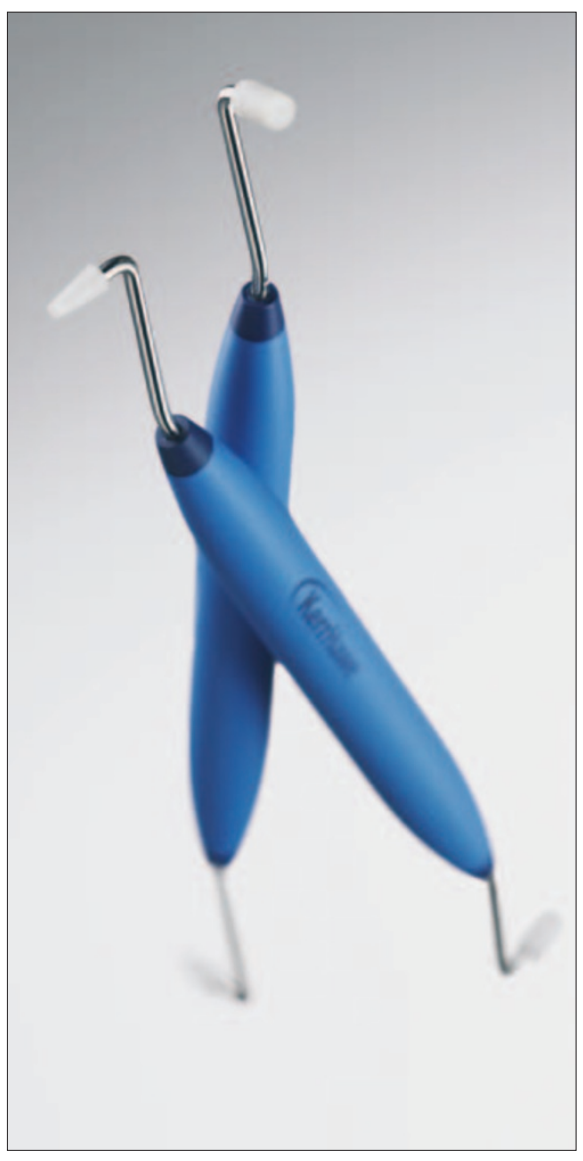

\section{TRADE NEWS \\ WHAT'S NEW}

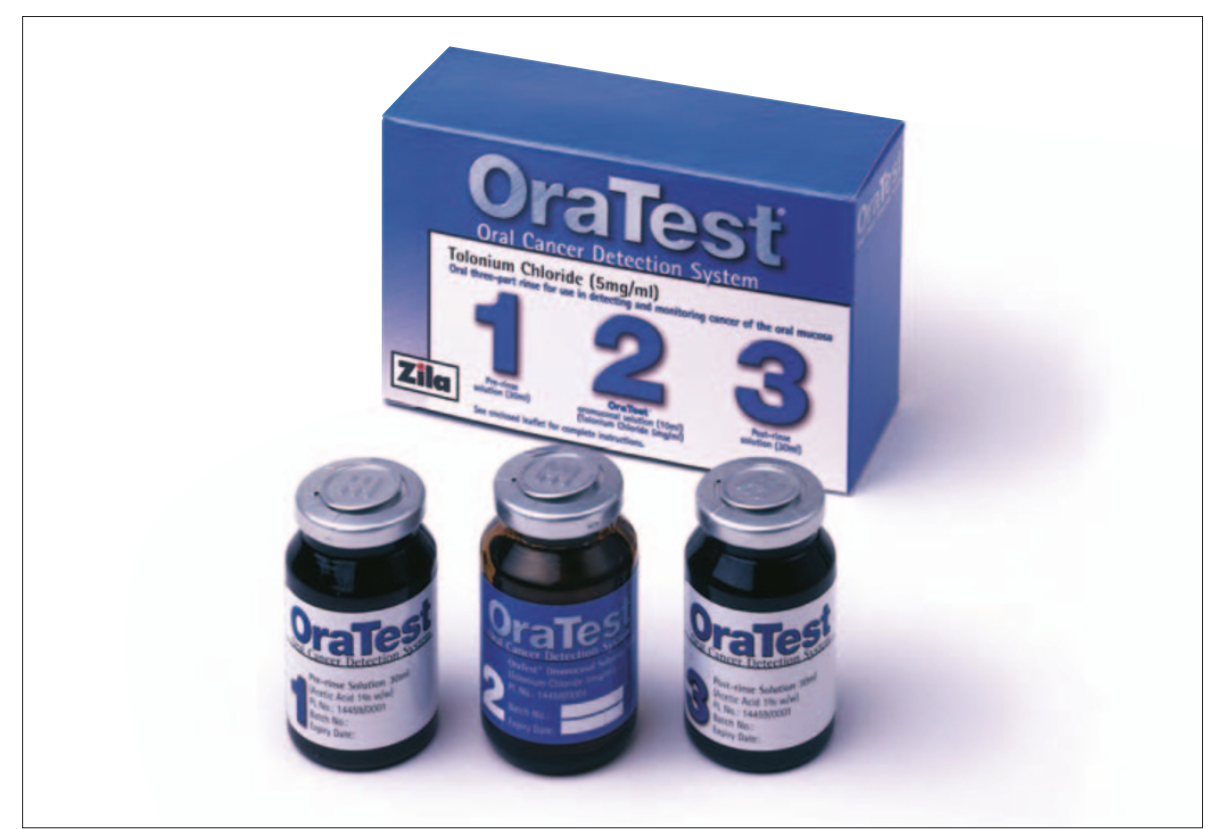

The OraTest mouth cancer detection system is used as an adjunct to a thorough head, neck and soft tissue exam in patients at risk for oral cancers. OraTest stains early, asymptomatic lesions a distinctive blue, highlighting the smallest potential malignancy and alerting the dentist to the need for further examination. In a two-step protocol, OraTest is reapplied 10-14 days later to alleviate false positives due to trauma or temporary inflammatory lesions. A positive stain at the second test mandates referral to a hospital specialist for biopsy.

Each kit is individually pre-packaged for single use, ensuring consistency, ease of administration, and uniform results. It is manufactured by Zila Inc.

Reader response number 51

\section{New dental anaesthetic launched}

Dentsply Ltd has announced the UK and Irish introduction of its new dental anaesthetic, Oraqix $25 / 25 \mathrm{mg}$ per $g$ periodontal gel (lidocaine, prilocaine). Oraqix is a non-injectable dental local anaesthetic indicated in adults for localised anaesthesia in periodontal pockets for diagnostic and treatment procedures such as probing, scaling and/or root planing. One of the products key benefits is that it is a liquid that turns to a gel at body temperature. This patented thermosetting property keeps it in place for effective absorption. Anaesthesia occurs quickly within 30 seconds, and there is no post-procedural numbness. It can be administered by means of a metric dental syringe or a specially designed Oraqix Dispenser.

Reader response number 52

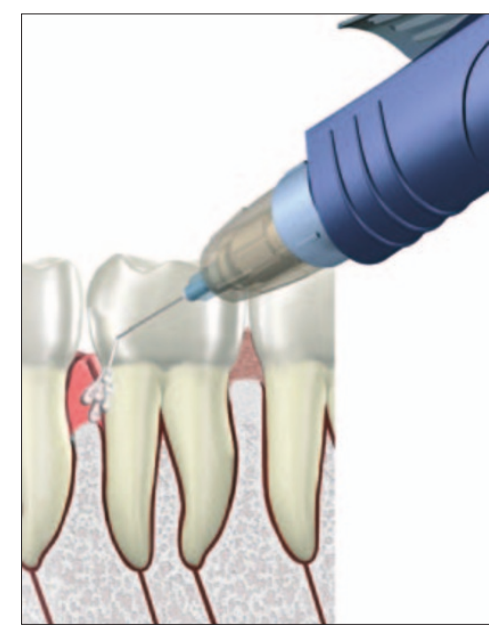



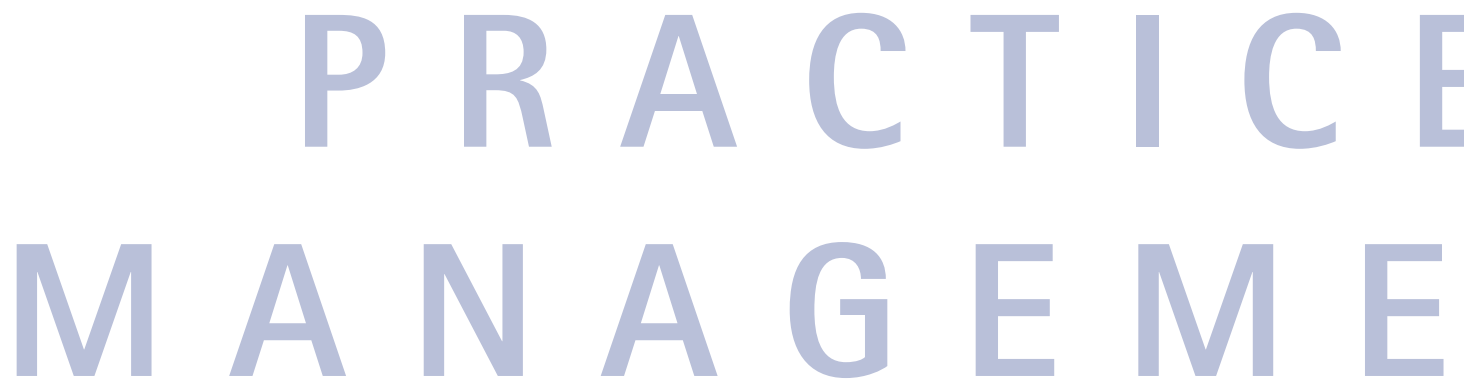

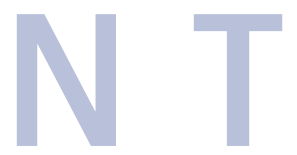

\section{Brightening up the practice}

Admor's range of reception furniture is available in a wide variety of colours, styles and designs, sympathetically complemented by interior accessories. There is a choice of straight or curved reception desks or modular reception units, which are durable, and are offered in many different finishes and patterns to suit all tastes and every practice. The company also supplies dentally themed accessories to brighten up even the darkest corners. Essential items such as signage and coat hooks, or tooth shaped lamps doubling as stools, wall clocks, clip boards, hand mirrors, desk tidies, pen holders and many more can be inviting to your patients.

Reader response number 53

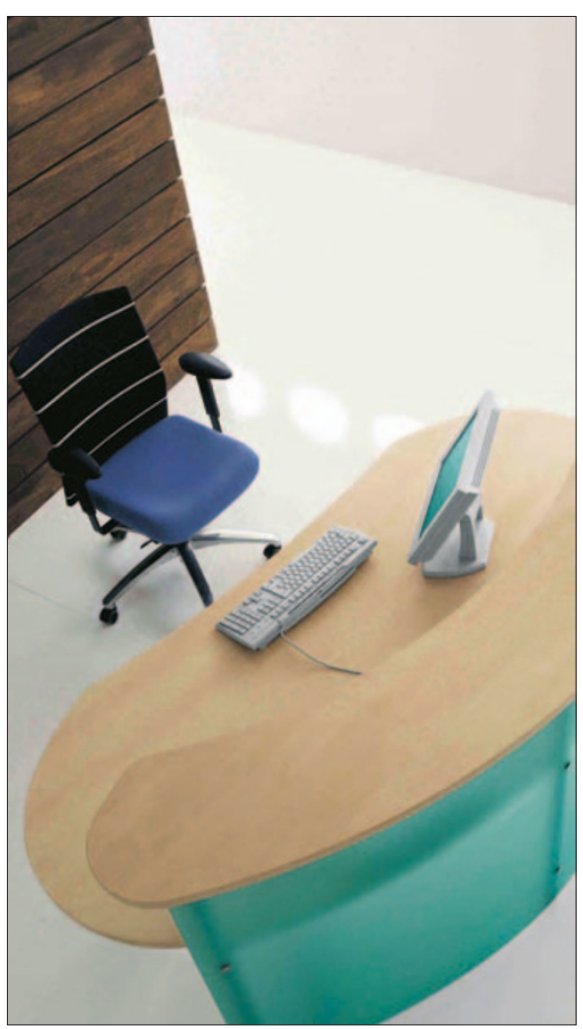

\section{Clearing up clutter}

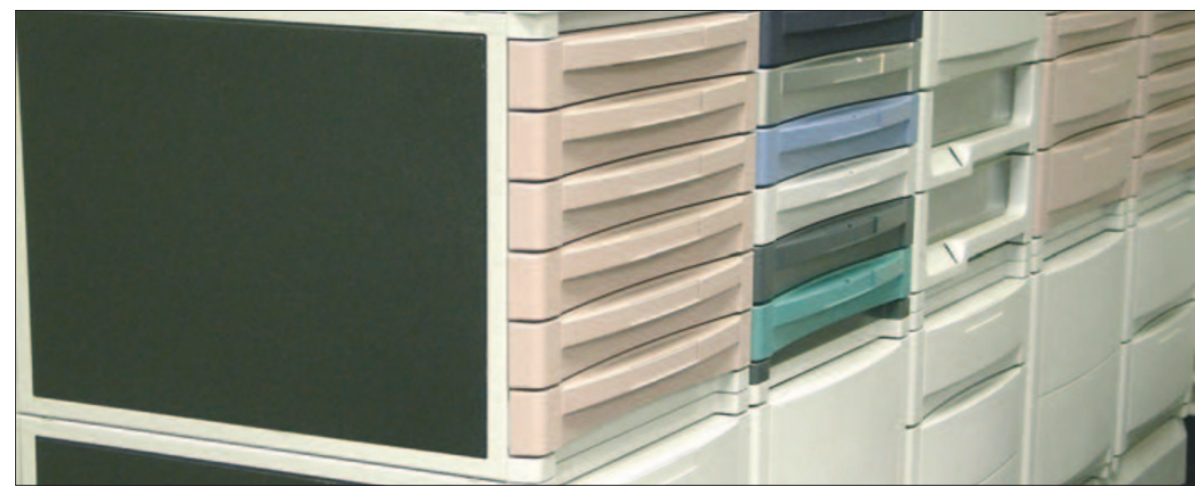

The VersaFile system has been adapted and expanded to suit a range of applications, including research, dental and medical labs. VersaFile, supplied by Hoare Laboratory Engineering Limited has a hi-tech appearance and offers state-of-the art storage.

The VersaFile concept is based upon a series of horizontal plastic drawers which fit neatly into a flexible modular drawer cabinet. Components are easily interchangeable and can be readily adapted to individual clients' requirements.

Hoare Laboratories have also just intro- duced the Highline range which offers versatility, as well as mobility. The mobile trolley units are available in nine formats, in a choice of two heights, and offer a range of different drawer sizes and storage options. Colour-coded drawer fronts can be used for ease of identification and, with a range of colours and finishes to choose from, the cabinets are guaranteed to fit into any working environment. The storage options can be tailored to meet the needs of the individual, which encourages users to put things back in their proper place. Reader response number 54

\section{Patient education software}

Clinical charting programme R4 from PracticeWorks is fully compatible with MEDiVision's Patient and Treatment Education programs, including CIC Explorer V9, PIC V9 and Mouth Modeller.

The time saving patient education tools enhance the patient's acceptance of dental treatments. The CiC Explorer V9 patient education tool illustrates treatments with high quality animations, while the Mouth Modeller V3 converts the practice management system's

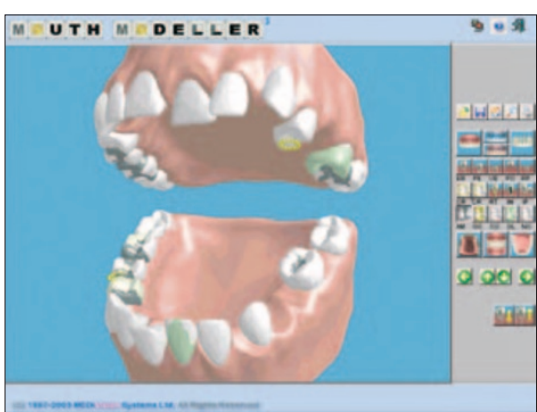
clinical chart into a pictorial computer model which rapidly conveys to the patient an accurate impression of a proposed treatment's cosmetic effect. Lastly, the PiC practice marketing programme is entertaining and informative, and can increase patient awareness of the possibilities in dentistry.

Reader response number 55 


\section{Versatile furniture solution}

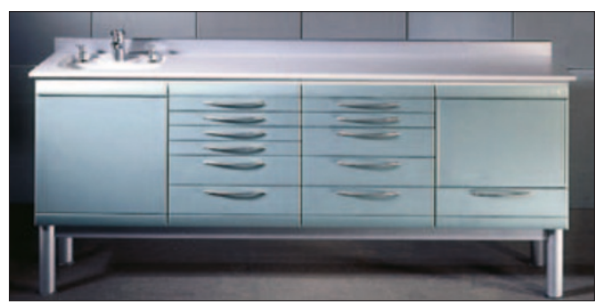

Paradigm Design, the dental furniture specialists and image creators have teamed up with Baisch to design a flexible and easy to install system. The Esprit range is freestanding and has the option of glass, Corian or Zodiac worktops. The simple storage system organises the many sundries and materials that are needed routinely and even greater flexibility is demonstrated by the ability to accommodate computers, keyboards, CPUs and special ancillary equipment. The unit offers nine different drawer arrangements and myriad drawer inserts.

Reader response number 56

\section{Durablity and style}

Henry Schein offers a range of cabinetry which has been developed to accommodate the wide differences between surgery locations. Each unit in each of Henry Schein's three ranges are individually manufactured in the UK to fit. The Praxis range has been designed to provide the dentist with a budget option without compromising on durability and quality and the Pacific range reflects attention to detail and incorporates hardwood (mahogany) dovetailed drawers with concealed self-closing runners. It has moulded drawer inserts and seamless Corian moulded work surfaces. The Penstock Range has over 500 different colour options, and is designed with flexibility in mind to meet the needs of dentists who want a wider choice when selecting cabinetry for their surgery layout.

All three Henry Schein cabinetry ranges take full account of the need for infection control, computerisation, and patient and dental team comfort.

Reader response number 57

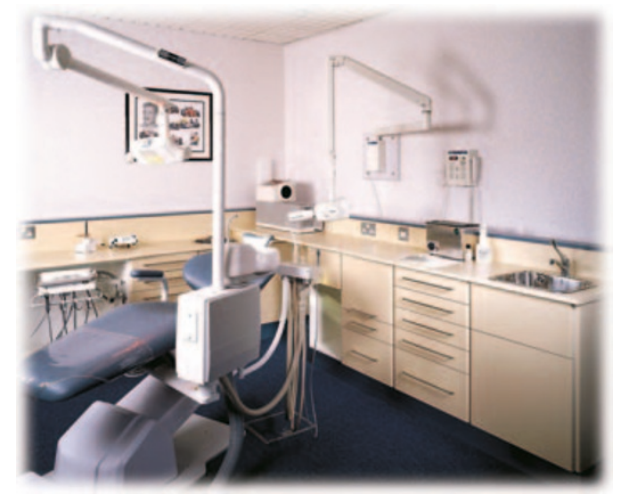

\section{Creating an integrated practice}

Kodak Dental Systems range of digital solutions can help create an integrated practice. Its practice management software features an appointment book, clear patient charting, an efficient reporting tool and a recall programme.

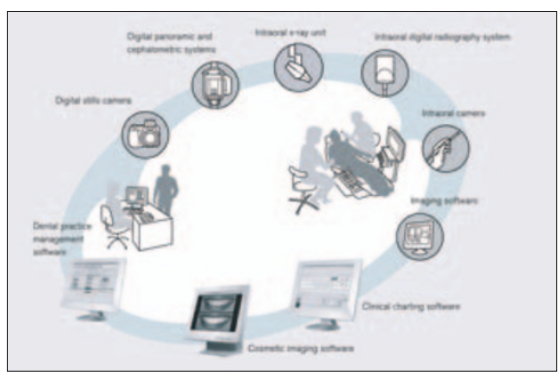

Its dentists' digital cameras incorporate a ring flash, a close-up lens, and the software to automatically export photos to patient records or a high quality printer while its digital intra-oral x-rays are high quality images with no need for film and chemicals.

The company's Panoral and Cephalometric x-ray have no film, no delay, and are fully compatible with other elements in the system while the Cosmetic imaging software is a new concept and enables digital photography to be introduced into dental imaging software to show the effect of tooth whitening and pre and post-treatment images.

The products have guaranteed compatibility with other imaging devices and practice management software, and come with a manufacturer's warranty.

Reader response number 58

\section{Tailored service}

Practice Plan has issued a new CD, "Your Practice, Your Future," which is a 20 minute CD that explains how Practice Plan will safeguard your current business and guarantee your future in dentistry. Introduced by John Tinsley, founder and Managing Director of Practice Plan, the benefits are clearly identified and reinforced by the experiences of practices already engaged with the company. A partnership with Practice Plan provides a custom-created, fully costed patient payment plan without compromising your practice's independence or individual needs of your patients. Your clinical freedom, choice of services and brand image are protected. Reader response number 59

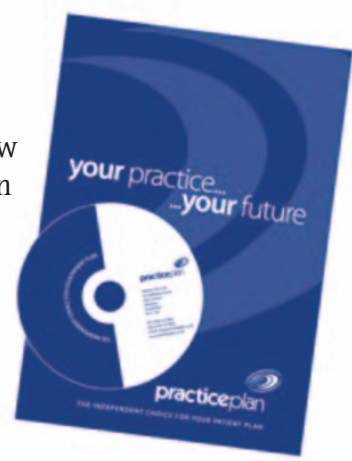

\section{New education programme}

Smile-on's "Key Skills in Primary Care” covers a significant spectrum of practice issues that can be applied in dental practices. The product is a CD ROM-based education programme using a scenario based learning methodology that covers seven specialist subject matters, to ensure the highest level of professionalism is maintained within your practice.

One such area covered is patient confidentiality in record keeping and suggests measures to ensure confidentiality. These measures are very much the commonsense approach, and reinforce practice protocols such as ensuring all filing cabinets are clearly labelled and secured, and computer monitors are kept out of the view of the general public. Dentists can ensure that patients records (including radiographs, $\mathrm{x}$-rays etc) are not left on display and discussions between staff of any patient should not take place where other patients could over-hear.

Reader response number 60

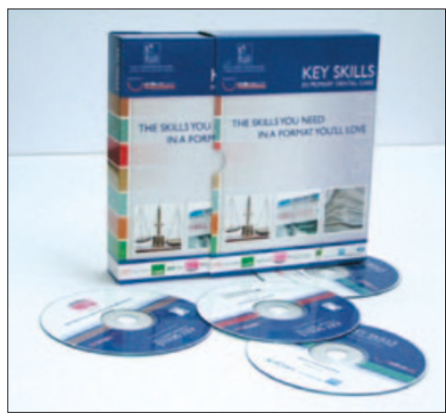

